\title{
Mild, Aqueous, Aerobic, Catalytic Oxidation of Methane to Methanol and Acetaldehyde Catalyzed by a Supported Bipyrimidinylplatinum-Polyoxometalate Hybrid Compound
}

\author{
Itsik Bar-Nahum, Alexander M. Khenkin and Ronny Neumann* \\ Department of Organic Chemistry, Weizmann Institute of Science, Rehovot, Israel, 76100
}

\section{Supplementary Material}

$\mathrm{Pt}$ (bipym) $\mathrm{Cl}_{2}$ : A solution of 2,2'-bipyrimidine (65 mg, $\left.0.41 \mathrm{mmol}, 1.14 \mathrm{eq}\right)$ in $5 \mathrm{~mL} \mathrm{MeOH}$ was added drop-wise to a suspension of $\mathrm{Pt}(\mathrm{DMSO})_{2} \mathrm{Cl}_{2}(150 \mathrm{mg}, 0.36 \mathrm{mmol}$, prepared according to Price, J. H.; Williamson, A. N.; Schramm, R. F.; Wayland, B. B. Inorg. Chem. 1972, 11, 1280) in $10 \mathrm{~mL} \mathrm{MeOH}$. After mixing for $12 \mathrm{~h}$ at RT the red precipitate was treated in an ultrasonic bath for $2 \mathrm{~h}$, washed with $\mathrm{EtOH}$ and ether before drying under vacuum. The yield was $140 \mathrm{mg}(92 \%$ yield). ${ }^{1} \mathrm{H}$ NMR $400 \mathrm{MHz}\left(\mathrm{DMSO}-d_{6}\right): \delta 8.00[\mathrm{t}, 2 \mathrm{H}(\mathrm{J}=4.97 \mathrm{~Hz})], \delta 9.35\left[\mathrm{dd}, 2 \mathrm{H}\left({ }^{1} \mathrm{~J}=4.75 \mathrm{~Hz}\right.\right.$, $\left.\left.{ }^{2} \mathrm{~J}=2.04 \mathrm{~Hz}\right)\right], \delta 9.69\left[\mathrm{dd}, 2 \mathrm{H},\left({ }^{1} \mathrm{~J}=6.5 \mathrm{~Hz},{ }^{2} \mathrm{~J}=1.97 \mathrm{~Hz}\right)\right] . \mathrm{IR}\left(\mathrm{cm}^{-1}\right): 677 \mathrm{~m}, 741 \mathrm{~s}, 818 \mathrm{~m}, 1029 \mathrm{~m}$, $1406 \mathrm{~s}, 1555 \mathrm{~m}, 1584 \mathrm{~s}, 3066 \mathrm{~m}$.

$\left[\mathrm{Pt}(\text { Mebipym }) \mathrm{Cl}_{2}\right]^{+} \mathrm{HSO}_{4}^{-}$: Dry Pt(bipym) $)_{2} \mathrm{Cl}_{2}(100 \mathrm{mg}, 0.24 \mathrm{mmol})$ and $\mathrm{Me}_{2} \mathrm{SO}_{4}(200 \mu 1,2.1$ mmol) were mixed in $15 \mathrm{~mL}$ anhydrous DMSO at $55^{\circ} \mathrm{C}$ for $15 \mathrm{~h}$ under Ar. The suspension cleared to a yellow solution after $1 \mathrm{~h}$. DMSO was removed by lyophilization and the resulting crude product was purified by addition of $\mathrm{EtOH}$. The precipitate was filtered, washed with EtOH and ether, and dried under vacuum. The yield was $100 \mathrm{mg}\left(78 \%\right.$ yield). ${ }^{1} \mathrm{H}$ NMR at $500 \mathrm{MHz}$ $\left(\mathrm{DMSO}-d_{6}\right): \delta 3.36(\mathrm{~s}, 3 \mathrm{H}), \delta 8.16[\mathrm{t}, 1 \mathrm{H},(\mathrm{J}=5.0 \mathrm{~Hz})], \delta 8.21[\mathrm{t}, 1 \mathrm{H},(\mathrm{J}=5.0 \mathrm{~Hz})], \delta 9.47[\mathrm{dd}$, $\left.1 \mathrm{H},\left({ }^{1} \mathrm{~J}=4.8 \mathrm{~Hz},{ }^{2} \mathrm{~J}=2.0 \mathrm{~Hz}\right)\right], \delta 9.49\left[\mathrm{dd}, 1 \mathrm{H},\left({ }^{1} \mathrm{~J}=5 \mathrm{~Hz},{ }^{2} \mathrm{~J}=2 \mathrm{~Hz}\right)\right], \delta 9.59\left[\mathrm{dd}, 1 \mathrm{H},\left({ }^{1} \mathrm{~J}=5.75 \mathrm{~Hz}\right.\right.$, $\left.\left.{ }^{2} \mathrm{~J}=2.0 \mathrm{~Hz}\right)\right], 9.89\left[\mathrm{dd}, 1 \mathrm{H},\left({ }^{1} \mathrm{~J}=6.0 \mathrm{~Hz},{ }^{2} \mathrm{~J}=3.0 \mathrm{~Hz}\right)\right] .{ }^{13} \mathrm{C} \mathrm{NMR}$ at $125.758 \mathrm{MHz}\left(\mathrm{DMSO}-d_{6}\right): \delta$ $52.77, \delta 124.51, \delta 124.69, \delta 155.29, \delta 156.28, \delta 160.64, \delta 160.86, \delta 161.37, \delta 162.56 \mathrm{ppm}$ MALDI-TOF MS (in $\left.\mathrm{CH}_{3} \mathrm{CN}\right): 536.73$ (100\%) (M=537.26).

$\left[\mathrm{Pt}(\text { Mebipym }) \mathrm{Cl}_{2}\right]^{+}\left[\mathrm{H}_{4} \mathrm{PV}_{2} \mathrm{Mo}_{10} \mathrm{O}_{40}\right]^{-}$on silica: A solution of $\left[\mathrm{Pt}(\mathrm{Mebipym}) \mathrm{Cl}_{2}\right]^{+} \mathrm{HSO}_{4}^{-}(25.9 \mathrm{mg}$, $48 \mu \mathrm{mol})$ in $100 \mathrm{~mL}$ acetonitrile was added slowly to a stirred solution of $\mathrm{H}_{5} \mathrm{PV}_{2} \mathrm{Mo}_{10} \mathrm{O}_{40}(113$ mg, $48 \mu \mathrm{mol}$ prepared according to Tsigdinos, G. A.; Hallada, C. J. Inorg. Chem. 1968, 7, 437441 ) in $200 \mathrm{~mL}$ acetonitrile. Anion exchange was clearly indicated by the very different solubilities of $\left[\mathrm{Pt}(\mathrm{Mebipym}) \mathrm{Cl}_{2}\right]^{+} \mathrm{HSO}_{4}^{-}$(water soluble) and $\left[\mathrm{Pt}(\mathrm{Mebipym}) \mathrm{Cl}_{2}\right]^{+}\left[\mathrm{H}_{4} \mathrm{PV}_{2} \mathrm{Mo}_{10} \mathrm{O}_{40}\right]^{-}$ (water insoluble). Silica gel (2.5 g, BDH-course silica) was added. The solvent removed by evaporation and the supported catalyst was dried under vacuum at $50{ }^{\circ} \mathrm{C}$ for $4 \mathrm{~h}$. The ${ }^{1} \mathrm{H}$ NMR and ${ }^{13} \mathrm{C}$ NMR spectra of $\left[\mathrm{Pt}(\mathrm{Mebipym}) \mathrm{Cl}_{2}\right]^{+}\left[\mathrm{H}_{4} \mathrm{PV}_{2} \mathrm{Mo}_{10} \mathrm{O}_{40}\right]^{-}$are identical to that of $\left[\mathrm{Pt}\left(\text { Mebipym) } \mathrm{Cl}_{2}\right]^{+} \mathrm{HSO}_{4}{ }^{-} \cdot{ }^{31} \mathrm{P} \mathrm{NMR}\right.$ at $101.27 \mathrm{MHz}\left(\mathrm{DMSO}-d_{6}\right): \delta-4.05, \delta-3.68, \delta-3.54, \delta$ $-3.25 \mathrm{ppm}$.

Analysis of the reaction mixture was by injection $1 \mu \mathrm{l}$ aliquots of the aqueous phase into a GCMS. Quantification was by use of 2-pentanol as external standard. 
${ }^{1} \mathrm{H}$ NMR of $\left[\mathrm{Pt}(\mathrm{Mebipym}) \mathrm{Cl}_{2}\right]^{+}\left[\mathrm{H}_{4} \mathrm{PV}_{2} \mathrm{Mo}_{10} \mathrm{O}_{40}\right]^{-}$in DMSO-d 6

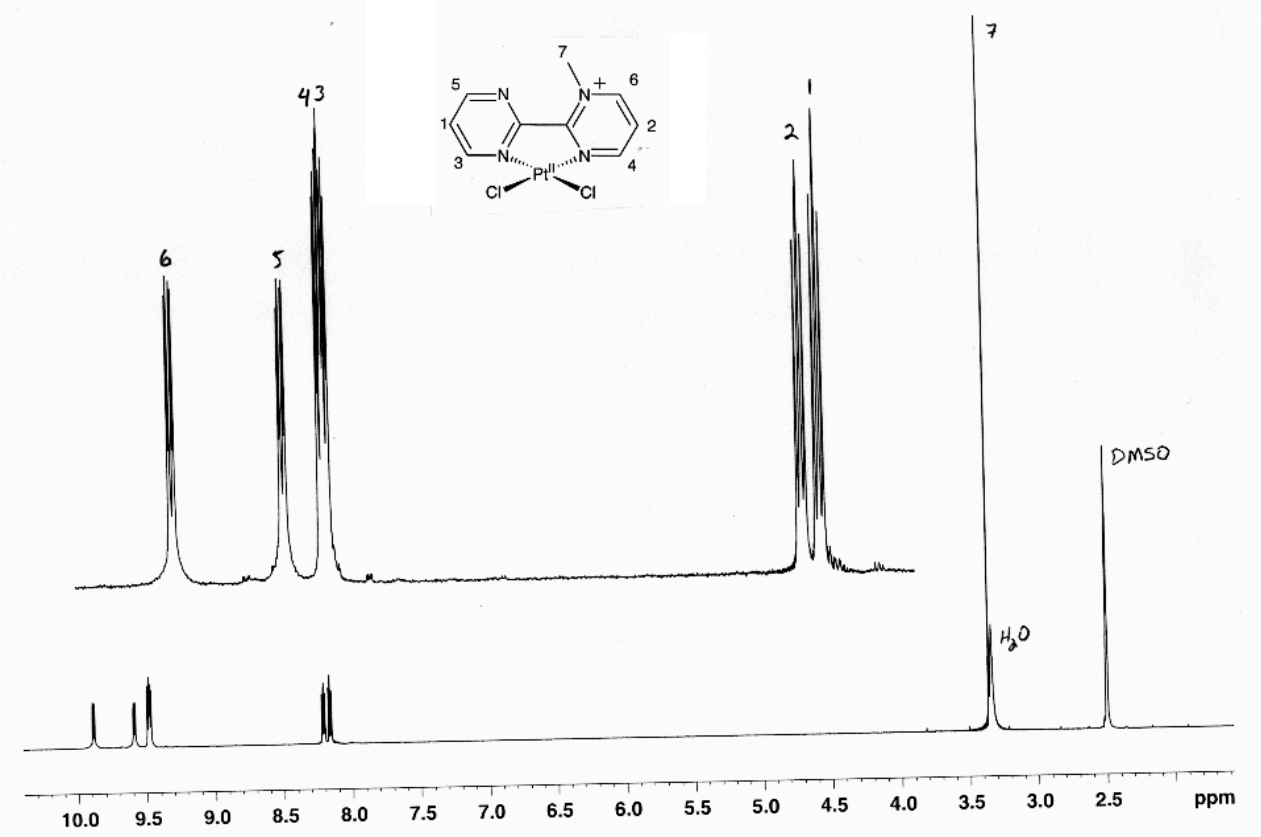

${ }^{13} \mathrm{C}$ NMR of $\left[\mathrm{Pt}\left(\text { Mebipym) } \mathrm{Cl}_{2}\right]^{+}\left[\mathrm{H}_{4} \mathrm{PV}_{2} \mathrm{Mo}_{10} \mathrm{O}_{40}\right]^{-}\right.$in DMSO-d6

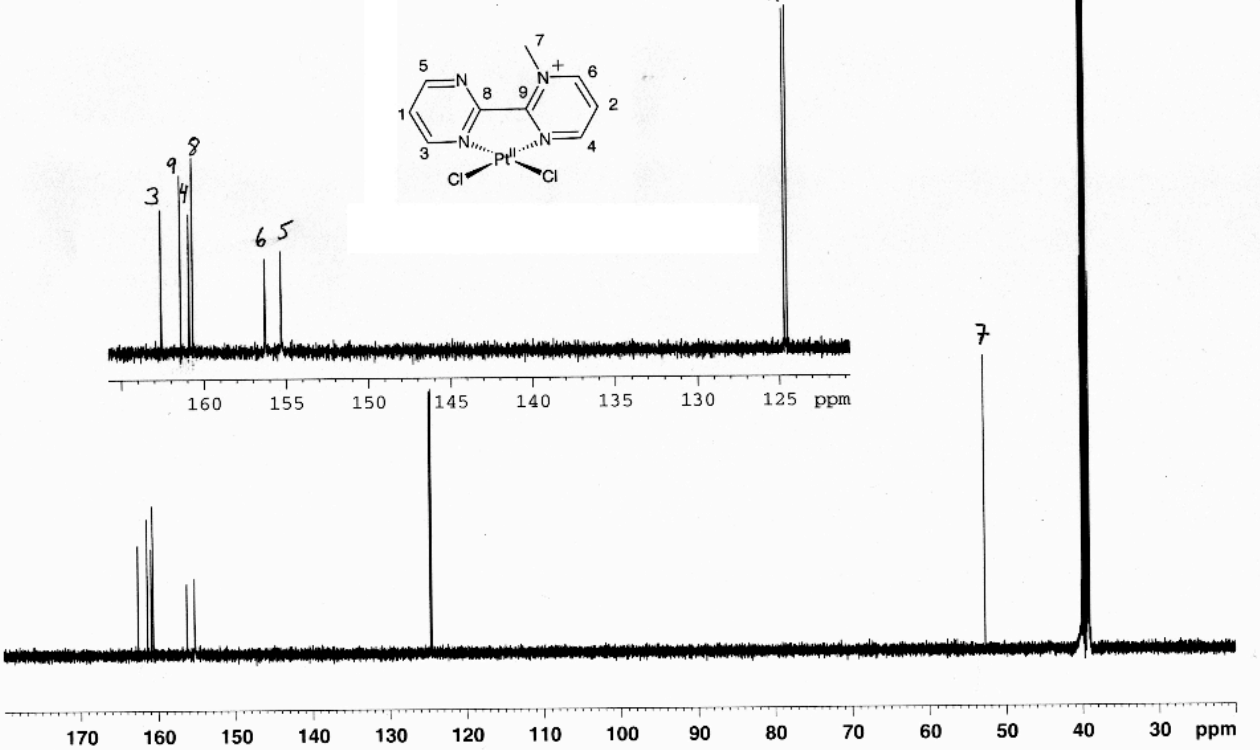


COSY NMR of $\left[\mathrm{Pt}\left(\text { Mebipym) } \mathrm{Cl}_{2}\right]^{+}\left[\mathrm{H}_{4} \mathrm{PV}_{2} \mathrm{Mo}_{10} \mathrm{O}_{40}\right]^{-}\right.$in DMSO-d6

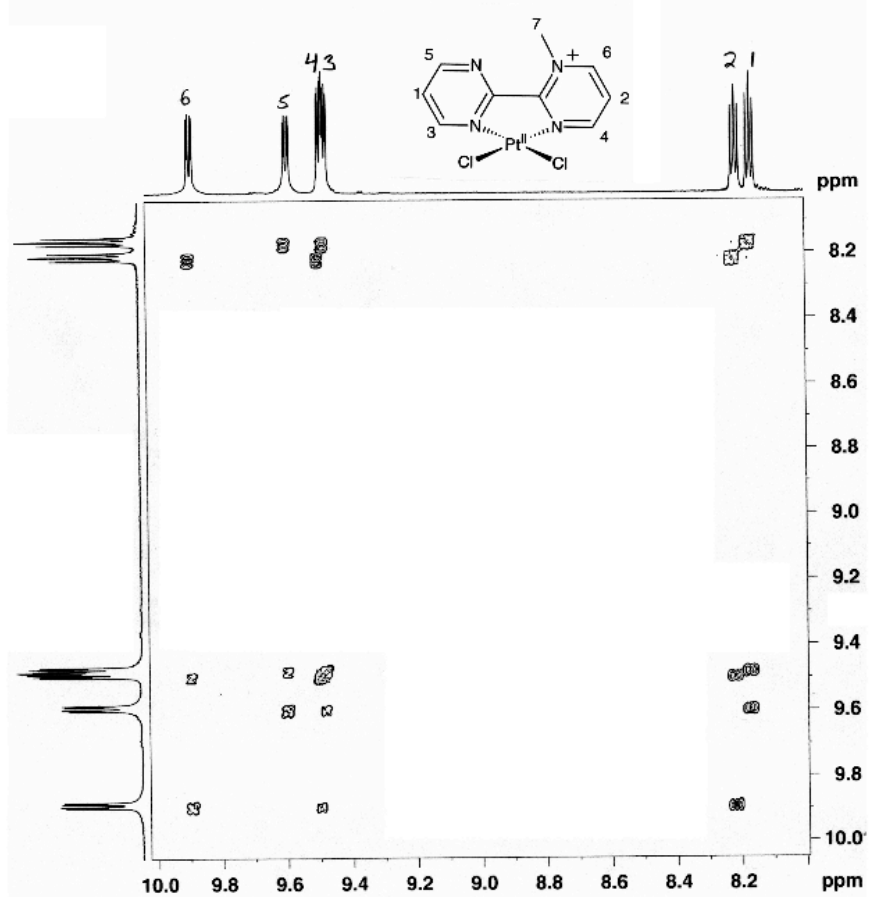

${ }^{1} \mathrm{H}_{-}{ }^{13} \mathrm{C}$ HMQC NMR of $\left[\mathrm{Pt}\left(\text { Mebipym) } \mathrm{Cl}_{2}\right]^{+}\left[\mathrm{H}_{4} \mathrm{PV}_{2} \mathrm{Mo}_{10} \mathrm{O}_{40}\right]^{-}\right.$in DMSO-d6
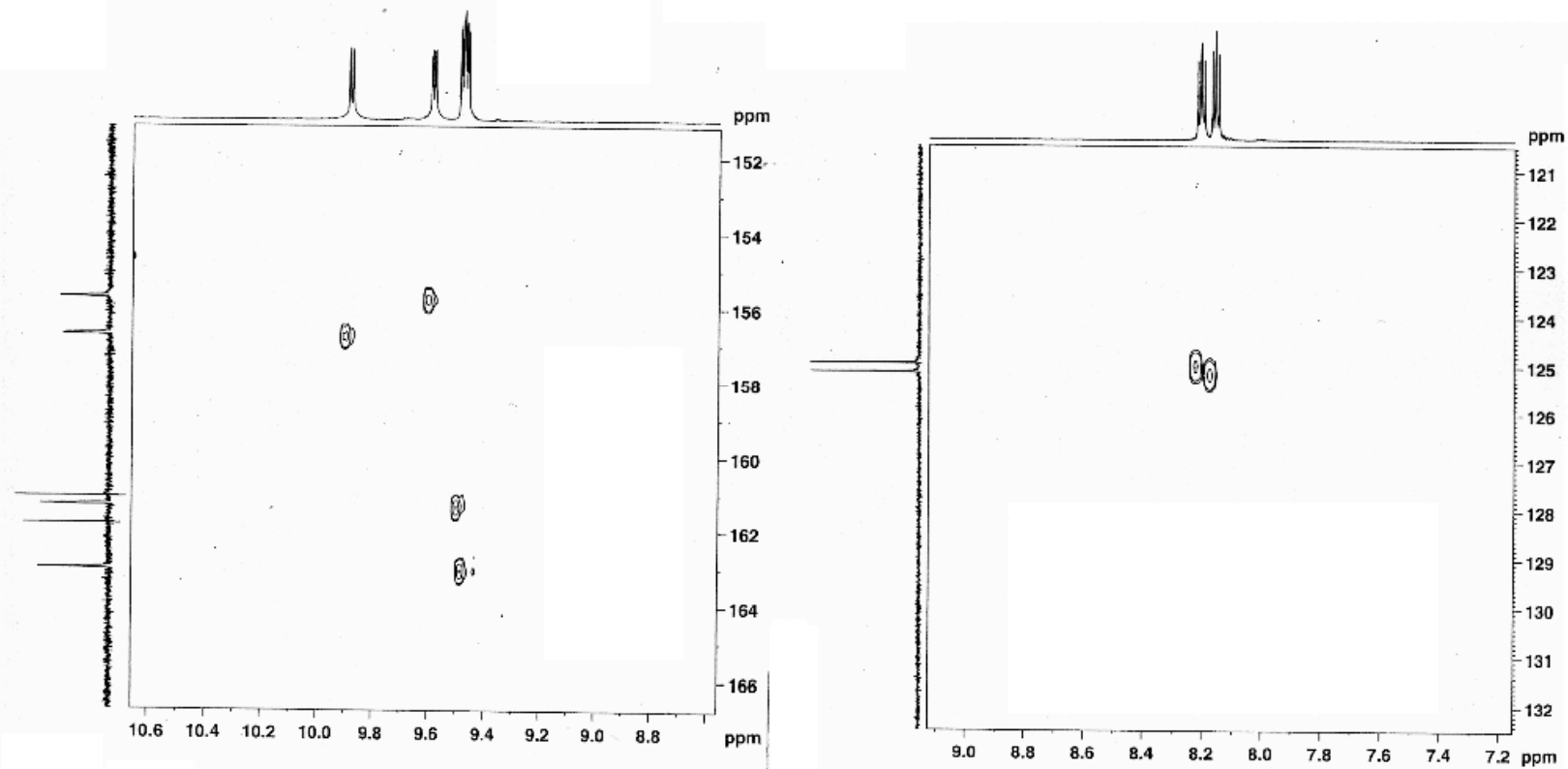
Figure 1S. Time-course profile for the oxidation of methane.

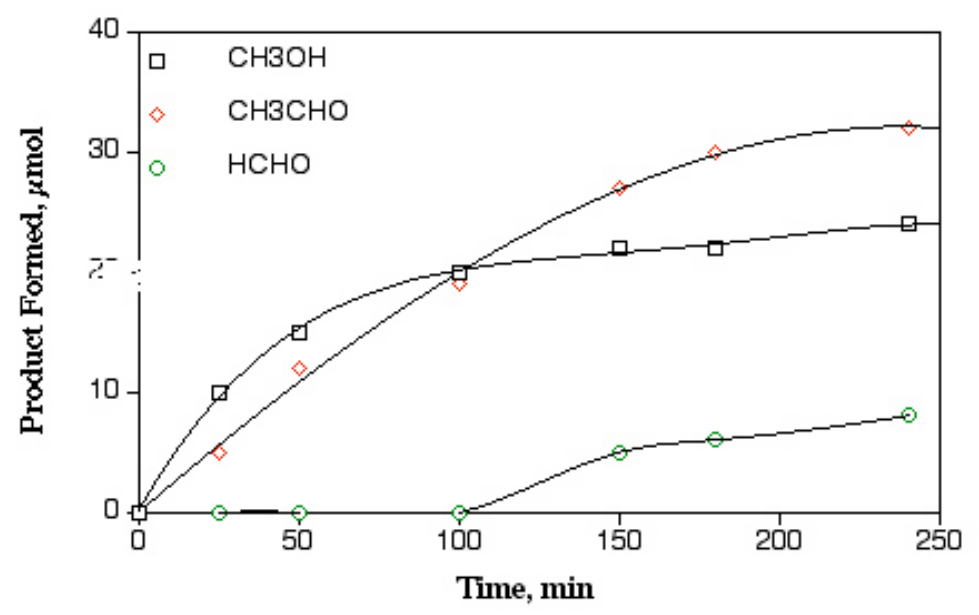

Reactions conditions: $2.88 \mu \mathrm{mol}$ catalyst, $2 \mathrm{~mL} \mathrm{H} 20$, $50 \mu \mathrm{mol} \mathrm{H} 2 \mathrm{SO} 4,30$ bar $\mathrm{CH} 4,2$ bar $02,50^{\circ} \mathrm{C}, 4 \mathrm{~h}$.

Figure 2S. Time-course profile for the oxidation of methanol and ethanol

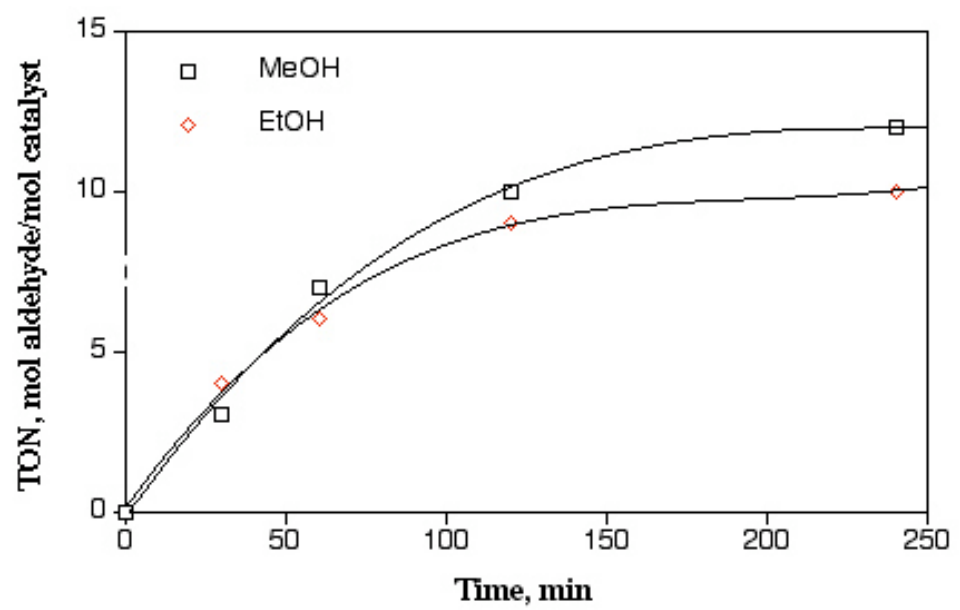

Reactions conditions: $2.88 \mu \mathrm{mol}$ catalyst, $2 \mathrm{~mL} \mathrm{H} 20$, $50 \mu \mathrm{mol} \mathrm{H} 2 \mathrm{SO} 4,1 \mathrm{mmol}$ alcohol, 2 bar $02,50^{\circ} \mathrm{C}, 4 \mathrm{~h}$. 